\title{
Vitamina D y salud ósea en la mujer posmenopáusica. Revisión
}

\section{Oscar Rosero Olarte}

Médico Especialista en Medicina Interna y Endocrinología

Universidad Militar Nueva Granada

Fiscal Médico Asociación Colombiana de Endocrinología, Diabetes y Metabolismo. Secretario Asociación Colombiana de Osteoporosis y Metabolismo Mineral ACOMM. Director Médico Instituto de Osteoporosis de los Llanos.

Fundador Asociación de Diabetes del Llano

Correspondencia:

Correo electrónico: oscarroseromd@yahoo.com

Conflicto de intereses:

El presente artículo no contó con fuentes de financiación externa. El autor ha sido conferencista para Farma de Colombia y Merck Sharp and Dome en temas de Vitamina D y ha participado en Advisory Board de Vitamina D para Sanofi.

\section{Resumen}

$\mathrm{L}$ a vitamina $\mathrm{D}$ es un determinante importante de la salud ósea y de la función neuromuscular. Recientemente se ha catalogado el déficit de vitamina D como un problema epidémico de proporción mundial.

Evidencia reciente sugiere que el aporte de vitamina $\mathrm{D}$ en dosis suficiente disminuye el riesgo de fracturas, así como el riesgo de presentar caídas en pacientes ancianos.

Mantener un nivel adecuado de vitamina D es una práctica simple y poco costosa que contribuye a disminuir las consecuencias que su déficit representa para la salud ósea.

Palabras clave: Vitamina D, osteoporosis, densidad mineral ósea, hiperparatiroidismo secundario, sarcopenia.

\section{Abstract}

Vitamin D is an important determinant of bone health and neuromuscular function. Vitamin D deficiency is now recognized as a worldwide epidemic.

Recent evidence suggests that vitamin D intake in a sufficient dose decreases the risk of fractures and falls among elderly patients.

It takes a simple and low-cost practice to maintain vitamin $D$ at suitable levels, thus contributing to decrease the consequences that its deficit entails for bone health.

Key Words: Vitamin D, osteoporosis, bone mineral density, secondary hyperparathyroidism, Sarcopenia.

\section{Introducción}

La vitamina D está ampliamente distribuida en la naturaleza, es fotosintetizada en muchas plantas y animales expuestos a luz solar. Su mayor papel en animales vertebrados y humanos es incrementar la absorción de calcio y fósforo para la mineralización del esqueleto. La deficiencia de vitamina D se manifiesta en los niños como raquitismo y en los adultos como osteomalacia $^{(1)}$.

Recientemente la vitamina D ha capturado la atención como un determinante de la salud ósea y la función neuromuscular, así mismo ahora se reconoce el déficit de vitamina $\mathrm{D}$ como un problema de proporciones epidémicas a nivel mundial $^{(2)}$. En los últimos 20 años se han publicado numerosos artículos con respecto al déficit de vitamina D y sus consecuencias en la población anciana, de la misma forma se puede asumir hoy en día que en el paciente anciano con osteoporosis, la debilidad muscular y las caídas pueden ser reflejo del déficit de vitamina D.

El objetivo de este artículo es revisar la prevalencia de la deficiencia de vitamina $\mathrm{D}$ en mujeres posmenopáusicas, sus consecuencias, prevención y tratamiento.

\section{Fisiología de la vitamina D y mineralización ósea}

La vitamina $\mathrm{D}$ se obtiene a partir de la dieta y por acción de la luz solar en la piel (figura 1). Durante la exposición solar, los fotones de luz ultravioleta B (UVB) penetran la epidermis y la dermis, donde son absorbidos por el 7-dehidrocolesterol que se encuentra en la membrana plasmática de estas células, el 7-dehidrocolesterol por acción de UVB se convierte a precolecalciferol que a su vez se convierte en colecalciferol, siendo lanzado fuera de la membrana plasmática al espacio extracelular donde se fija a la proteína transportadora de vitamina D $\left(\mathrm{DBP}^{*}\right)^{(2)}$.

El primer paso en la activación metabólica de la vitamina D es la hidroxilación del carbono 25 , que ocurre principalmente en el hígado a través del citocromo p-450, el segundo paso en la bioactivación de la vitamina $\mathrm{D}$ es la formación de $1 \alpha-25$ hidroxivitamina $\mathrm{D}\left(1,25(\mathrm{OH})_{2} \mathrm{D}\right)$ a partir de la 25-hidroxivitamina $\mathrm{D}$, proceso que ocurre bajo condiciones fisiológicas en el riñón ${ }^{(3)}$. 
La 1 $\alpha, 25$-hidroxivitamina D interactúa con el receptor nuclear de vitamina $\mathrm{D}\left(\mathrm{VDR}^{*}\right)$ que a su vez se une al receptor de ácido retinoico, formando un complejo que es reconocido por secuencias genéticas específicas conocidas como elementos de respuesta a vitamina D (VDRE*), para abrir la información genética responsable de sus acciones biológicas ${ }^{(4)}$.

En el intestino, la 1,25(OH) 2 D induce la expresión de un canal epitelial de calcio, una proteína fijadora de calcio (calbindina) y una variedad de otras proteínas que ayudan a transportar el calcio de la dieta a la circulación ${ }^{(4)}$, también incrementa el transporte activo de fósforo a través de la estimulación de la expresión del cotransportador Na-P y cambios en la composición de la membrana del enterocito que incrementan la fluidez y el transporte de fosfato ${ }^{(5)}$.

La vitamina $\mathrm{D}$ es esencial para el desarrollo y mantenimiento del esqueleto mineralizado, estimulando a los osteoblastos para producir osteocalcina y fosfatasa alcalina, lo que indica un proceso activo de osteogénesis ${ }^{(6)}$ y a su vez la 1,25 $(\mathrm{OH})$ 2D también interactúa con su VDR en el osteoclasto estimulando la expresión del ligando de unión al receptor activador de NF-kB (RANKL) ${ }^{(7)}$, regulando de esta forma tanto la formación como el remodelado óseo, proceso dinámico requerido para una adecuada salud ósea.
Figura 1. Producción cutánea de vitamina D y su metabolismo. Durante la exposición solar el 7-dehidrocolesterol (7-DHC) en la piel absorbe la radiación solar UVB y es convertido a precolecalciferol (PRE-D3), y este bajo efecto del calor sufre transformación a colecalciferol. La vitamina D que ingresa con la dieta o por transformación cutánea entra a la circulación y es transformada en el hígado por acción de la 25-hidroxilasa en 25-hidroxivitamina D 3 (25(OH) D3), esta entra nuevamente a la circulación y a nivel renal se convierte a 1,25 -dihidroxivitamina $D$ 3 (1,25-(OH) 2D3) por acción de la $1 \alpha$-hidroxilasa. La 1,25-(OH) 2D3 ejerce su efecto sobre diversos órganos, regulando el metabolismo del calcio.
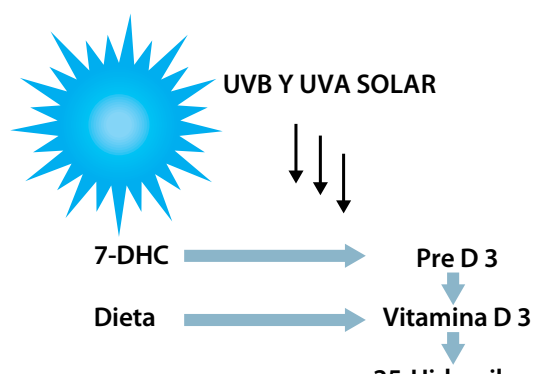

25-Hidroxilasa

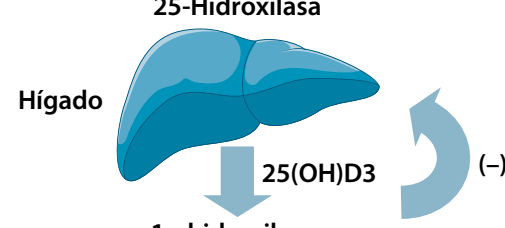

$1 \alpha$ hidroxilasa

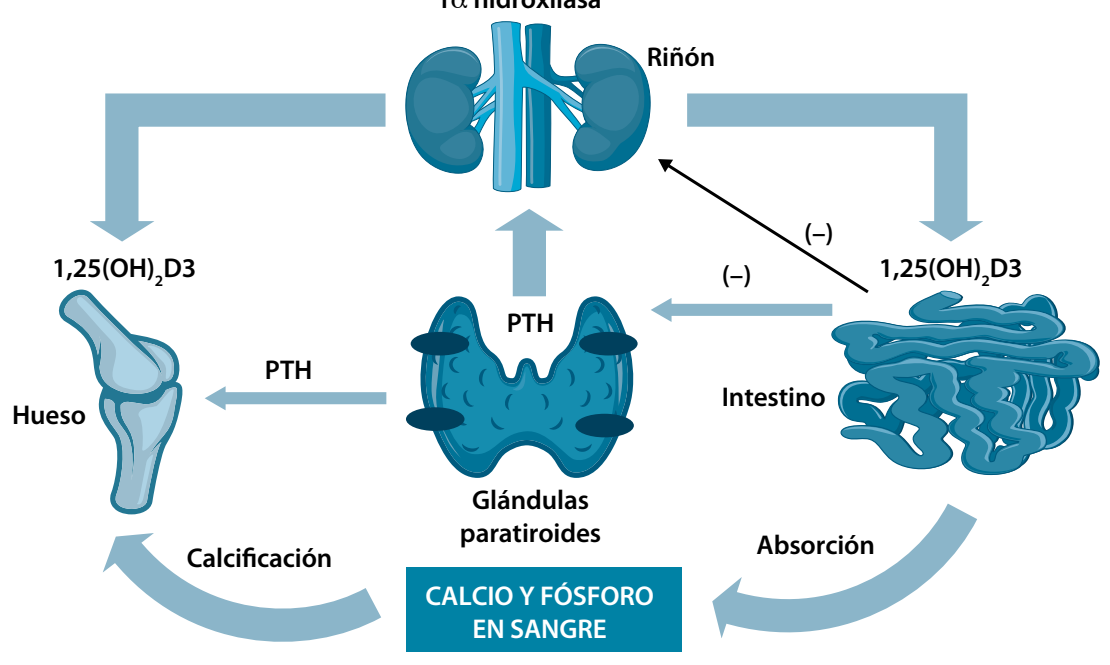

\section{Cambios en el metabolismo} de la vitamina $\mathrm{D}$ con la edad

La eficiencia en la producción de vitamina D en la piel disminuye con la edad; sin embargo, la absorción continua siendo adecuada incluso en edad avanzada ${ }^{(8)}$.

La hidroxilación hepática de la vitamina D se preserva con la edad pero puede estar comprometida por enfermedades hepáticas, no ocurre así con la $1 \alpha$-hidroxilación renal que puede disminuir con la edad, paralela a la disminución en la función renal(6).

La disminución de la absorción de calcio se ha atribuido a una disminución en los niveles séricos de 1,25-(OH) 2D. Igualmente, con la edad disminuye la DBP así como la albúmina ${ }^{(4)}$.

\section{Causas de deficiencia de Vitamina D}

La tabla 1 muestra las causas más frecuentes del déficit de vitamina D.

\section{Consecuencias del déficit de vitamina $D$ para la salud ósea}

Bajas concentraciones de vitamina D llevan a disminución en la absorción del calcio que conduce a un incremento en la secreción de PTH que estimula la producción de 1,25- $(\mathrm{OH}) 2 \mathrm{D}$, lo que se denomina "hiperparatiroidismo secundario". Este incremento en la PTH causa aumento en el recambio óseo con el objetivo de movilizar los depósitos de calcio óseo para mantener la homeostasis 
Tabla 1. Causas de déficit de vitamina $D$

Déficit de ingesta o absorción

1. Baja Ingesta

2. Inadecuada exposición solar

Malabsorción

1. Gastrectomía - Cirugía bariátrica

2. Síndromes de malabsorción: fibrosis quística, enfermedad de Crohn, enteritis por radiación, enfermedad inflamatoria intestinal

Defectos en la 25 - Hidroxilación

1. Falla hepática

2. Fármacos: anticonvulsivantes, antituberculosos, glucocorticoides, antimicóticos, medicaciones para VIH

Defectos en la 1-a -25 Hidroxilación

1. Insuficiencia renal

2. Hipoparatiroidismo

3. Raquitismo resistente a vitamina $D$ tipo I

4. Raquitismo hipofosfatémico

5. Osteomalacia tumoral

Falla en la respuesta al calcitriol

1. Raquitismo resistente a vitamina D tipo II

Modificado de J Clin Endocrinol Metab. 2011, 96(7):1911-1930

del metabolismo del calcio, llevando a la consiguiente pérdida de masa ósea ${ }^{(6)}$.

El hiperparatiroidismo secundario se ha propuesto como el principal mecanismo por el cual el déficit de vitamina D predispone a fracturas de cadera, habiéndose demostrado que el incremento de PTH en pacientes ancianos se asocia con déficit de vitamina $\mathrm{D}^{(9)}$. Esta relación inversa entre 25-(OH) vitamina D y PTH se ha observado no sólo en ancianos sino también en mujeres posmenopáusicas con edades entre 45 y 65 años ${ }^{(10)}$.

Se han publicado muchos reportes sobre la relación entre osteomalacia y fractura de cadera. En el estado de déficit de vitamina D, el nuevo osteoide formado no puede ser adecuadamente mineralizado, produciendo osteomalacia. Aunque la osteoporosis es una enfermedad silente hasta que ocurren las fracturas, la osteomalacia se asocia con dolor óseo que puede ser diagnosticado como fibromialgia, miosisits o síndrome de fatiga crónica ${ }^{(11)}$. La causa de este dolor es que el osteoide no mineralizado se hidrata y provee poco soporte para fibras sensoriales en el periostio ${ }^{(12)}$.

Numerosos estudios han establecido una relación directa entre la concentración sérica de 25-(OH) D y la densidad mineral ósea (DMO) en cadera; la correlación fue positiva cuando los niveles séricos de 25-(OH) D se encontraron por debajo de $30 \mathrm{ng} / \mathrm{mL}^{(13)}$.

Otra interpretación de la asociación entre bajas concentraciones de 25-(OH) D y baja DMO es el estilo de vida sedentario, éste puede causar pérdida ósea por inmovilidad y poca exposición a luz solar ${ }^{(6)}$. De la misma forma se han podido correlacio- nar los niveles bajos de vitamina D sérica con la presencia de fracturas osteoporóticas. En un estudio de fracturas osteoporóticas realizado en cuatro centros de Estados Unidos, los niveles de $25-(\mathrm{OH})$ D por debajo de 22,8 $\mathrm{ng} / \mathrm{mL}$ se asociaron con un incremento del riesgo de fractura (RR 2,1 ajustado para edad y peso) de la misma forma se pudo determinar que el $50 \%$ de las pacientes tenían niveles de $25-(\mathrm{OH}) \mathrm{D}<30 \mathrm{ng} / \mathrm{mL}^{(14)}$. En Noruega, en un estudio con 21.774 hombres y mujeres con seguimiento durante 10 años, se encontró una relación inversa entre los niveles de $25(\mathrm{OH})$ D y el riesgo de fractura de cadera, determinando que los pacientes con niveles por debajo de 16,8 ng/mL tenían 38\% más de riesgo de fractura de cadera al compararlos con los que tenían niveles superiores a $27,1 \mathrm{ng} / \mathrm{mL}^{(15)}$.

La sarcopenia es un síndrome caracterizado por una progresiva y generalizada pérdida de masa de músculo esquelético así como de su fortaleza, con un riesgo incrementado de eventos adversos como limitación física, pobre calidad de vida y muerte ${ }^{(16)}$. La marcada disminución de la fuerza muscular en los ancianos se relaciona con pérdida de masa muscular, menos músculo, menos fuerza. Con la edad también cambia el metabolismo de la vitamina $\mathrm{D}$, entre los 75 y 80 años disminuye la producción de 1-25 $(\mathrm{OH}) 2 \mathrm{D}$, por pérdida de función renal, afectando el transporte de calcio al interior de los mús$\operatorname{culos}^{(17)}$. La vitamina D ejerce efectos rápidos y genómicos en las células musculares. Estos efectos se relacionan con el manejo del calcio intracelular, la diferenciación y la composición de las proteínas musculares contráctiles ${ }^{(18)}$.

\section{Diagnóstico y prevalencia de la deficiencia de vitamina $D$}

La hidroxilación de la vitamina D hepática está pobremente regulada, mientras que la actividad de la $1 \alpha$-hidroxilasa es altamente regulada por el calcio ingerido directamente a través de cambios en el calcio sérico o indirectamente alterando los niveles de hormona paratiroidea (PTH). De esta forma, los niveles de 25-(OH) D se incrementan en proporción al ingreso de vitamina $\mathrm{D}$, por esta razón los niveles plasmáticos de 25$(\mathrm{OH})$ D se usan comúnmente como un indicador del estatus de vitamina $\mathrm{D}^{(3)}$.

Aunque no hay consenso con respecto a los niveles séricos óptimos de 25-(OH) D, la deficiencia es definida por muchos expertos como un nivel por debajo de $20 \mathrm{ng} / \mathrm{mL}$ (50 nmol/L) ${ }^{(19)}$. Sin embargo, esto depende de variables como el clima, la exposición solar y los hábitos de vestido, que determinan variaciones entre países.

Un nivel de $25-(\mathrm{OH})$ D de 21 a $29 \mathrm{ng} / \mathrm{mL}$ (52 a $72 \mathrm{nmol} / \mathrm{l}$ ) puede ser considerado como insuficiencia relativa de vitamina $\mathrm{D}$ y un nivel por encima de $30 \mathrm{ng} / \mathrm{mL}$ se puede considerar como adecuado ${ }^{(20)}$.

Con base en estas definiciones se estima que más de 1.000 millones de personas alrededor del mundo presentan deficien- 
cia o insuficiencia de vitamina $\mathrm{D}(2)$, y más del $50 \%$ de mujeres posmenopáusicas que toman medicación para osteoporosis tienen niveles subóptimos de vitamina $\mathrm{D}(<30 \mathrm{ng} / \mathrm{mL})^{(21)}$.

En un estudio al final del invierno, el $42 \%$ de mujeres entre 19 y 45 años en Estados Unidos tenían niveles de 25- $(\mathrm{OH}) \mathrm{D}$ por debajo de $20 \mathrm{ng} / \mathrm{mL}^{(22)}$, en otro estudio chileno con 555 mujeres posmenopáusicas se encontró que el $47,5 \%$ de las pacientes estudiadas presentaban hipovitaminosis $\mathrm{D}^{(23)}$.

Las personas que viven cerca al Ecuador que se exponen a la luz solar sin protector solar, usualmente presentan niveles de 25- $(\mathrm{OH})$ D por encima de $30 \mathrm{ng} / \mathrm{mL}$. Sin embargo, en un estudio realizado en Hawai entre personas jóvenes sanas con exposición solar en promedio de $28,9 \mathrm{~h} /$ semana se encontró un valor promedio de $25-(\mathrm{OH}) \mathrm{D}$ de $31,6 \mathrm{ng} / \mathrm{mL}$ y usando como punto de corte $30 \mathrm{ng} / \mathrm{mL}$, el $51 \%$ de esta población tenía bajos niveles de vitamina $\mathrm{D}^{(24)}$.

En Colombia se han publicado tres estudios en relación con los niveles de vitamina D en mujeres posmenopáusicas, uno realizado en la ciudad de Bogotá, encontró niveles por debajo de $32 \mathrm{ng} / \mathrm{ml}$ en el 69,5\% de las pacientes estudiadas ${ }^{(25)}$, otros dos realizados en Medellín mostraron una prevalencia de insuficiencia de vitamina D de $55,1 \%^{(26)}$ y $77 \%{ }^{(27)}$, lo que indica que el problema del déficit de vitamina D no sólo afecta países con baja exposición solar, sugiriendo la importancia de una adecuada determinación y suplementación en nuestras mujeres en la posmenopausia.

\section{Prevención y tratamiento}

La deficiencia de vitamina $\mathrm{D}$ puede ser tratada con exposición solar o irradiación UV, incremento del consumo de la vitamina $\mathrm{D}$ en la dieta o con suplementos orales o parenterales.

Los resultados deben ser valorados con base en el incremento de los niveles séricos de 25- $(\mathrm{OH}) \mathrm{D}$, en la disminución de la PTH o la disminución de los marcadores de remodelado óseo. Como la 25-(OH) D posee una vida media prolongada, los resultados sólo pueden ser determinados de 3 a 6 meses después de iniciada la terapia ${ }^{(6)}$.

Exposición solar y radiación ultravioleta B: La exposición de los brazos y piernas durante 5 a 30 minutos entre las 10 a.m. y las 3 p.m. 2 veces a la semana es frecuentemente adecuada ${ }^{(28)}$. Sin embargo, la Asociación Americana de Dermatología desde el año 2009 no recomienda la exposición solar como método para obtener vitamina $\mathrm{D}$ por el riesgo de cáncer de piel derivado de la exposición solar sin protección adecuada ${ }^{(29)}$.

\section{Suplementacion oral}

Efecto de la vitamina $D$ en la producción de hormona paratiroidea: En las personas ancianas con deficiencia de vitamina D los niveles séricos de PTH usualmente disminuyen después de la suplementación con vitamina D. En un estudio con pacientes psicogeriátricos con niveles basales de 25- $(\mathrm{OH})$
D de 8 ng/mL la PTH disminuyó 30\% después de la suplementacion o irradiación UV (30). Una disminución similar aunque superior, en este caso cercana al 50\% fue observada en $72 \mathrm{mu}-$ jeres ancianas con deficiencia de vitamina $\mathrm{D}$ tras la suplementación con vitamina D 3 (800 UI/d) y calcio $(1000 \mathrm{mg} / \text { día })^{(31)}$ y más recientemente se demostró una reducción significativa de la PTH tanto en administración parenteral como oral en pacientes mayores de 65 años ${ }^{(32)}$.

\section{Efectos en recambio óseo}

La disminución en la función paratiroidea producida por los suplementos de vitamina D es seguida por una disminución en los marcadores de remodelado óseo. La combinación de vitamina D y calcio tiene mayor efecto suprimiendo el recambio óseo. La suplementación durante un año de vitamina D3, tanto en dosis de 800 UI como en dosis de 6.500 UI, fue seguida de una disminución del 50\% del N-Telopéptido urinario, una disminución de $20 \%$ en la osteocalcina sérica y una disminución de $10 \%$ en la fosfatasa alcalina ósea ${ }^{(33)}$.

\section{Efectos sobre la densidad mineral ósea (DMO)}

La terapia con vitamina D puede incrementar la DMO en varias regiones del esqueleto. En un estudio se compararon dosis de vitamina D de 100 UI y 400 UI/día en mujeres posmenopáusicas; el grupo con dosis altas mostró menor pérdida ósea en cuello femoral que el grupo de dosis bajas $(-1,0 \%$ vs $-2,5 \%, p<0,01)^{(34)}$. Un metanálisis recientemente publicado mostró un pequeño beneficio en mejoría de la densidad mineral ósea en cuello femoral (diferencia media ponderada de $0,8 \%, 95 \%$, IC $0,2-1,4$ ) lo que sugiere mayor efecto sobre el hueso cortical que en el trabecular ${ }^{(35)}$.

\section{Efecto sobre la fuerza muscular}

La debilidad muscular proximal es síntoma de osteomalacia, la suplementación con vitamina D puede mejorar la fuerza muscular con disminución en la incidencia de caídas. Un metanálisis de cinco estudios doble ciego, aleatorizados, controlados que involucró a 1.237 individuos ancianos (edad promedio 70 años) tratados con varios análogos de vitamina D durante un periodo de 2 meses a 3 años, indicó que el tratamiento con vitamina D reduce el riesgo de caída en $22 \%$ comparado con placebo o tratamiento con calcio solo. El número necesario a tratar (NNT) fue de 15 o el equivalente a tratar 15 pacientes con vitamina D durante un año para prevenir la caída en una persona $^{(36)}$. Otro metanálisis, esta vez con 45.782 pacientes, demostró que la suplementación con vitamina $\mathrm{D}$ reduce el riesgo de caídas de forma significativa; sin embargo, este beneficio no se evidenció sin la coadministración de calcio suplementario ${ }^{(37)}$.

\section{Efecto en el riesgo de fractura}

Aproximadamente el 33\% de las mujeres entre 60 y 70 años tienen osteoporosis y el $66 \%$ a los 80 años. Se estima 
que el $47 \%$ de las mujeres mayores de 50 años pueden presentar una fractura osteoporótica en algún momento de la vida restante ${ }^{(38)}$. En Colombia, la prevalencia e incidencia de la osteoporosis se desconoce debido al subregistro diagnóstico, la cifra estimada de egresos hospitalarios por osteoporosis en nuestro país es cercana al $0,02 \%{ }^{(38)}$.

Un metanálisis evaluó los resultados de la suplementación con vitamina D en el riesgo de fractura, en él se evaluaron cinco estudios aleatorizados sobre el riesgo de fractura de cadera $(n=9.294)$ y 7 estudios que valoraban el riesgo de fractura no vertebral $(n=9.820)$. Una dosis de 700-800 UI/día de vitamina $\mathrm{D}$ redujo significativamente el riesgo de fractura de cadera en $26 \%$ y el riesgo de fractura no vertebral en $23 \%$, sin que se observara beneficio significativo con dosis de 400 UI/día, postulando que dosis de menos de 700 UI día no tienen efecto sobre riesgo de fractura ${ }^{(39)}$.

Un metanálisis más reciente que incluyó 11 estudios doble ciego, aleatorizados, con 31.022 personas mayores de 65 años, también encontró mayor tasa de reducción del riesgo de fracturas de cadera y otros sitios con dosis entre 792 y 2000 UI/día ${ }^{(40)}$.

\section{Dosis óptimas de vitamina D}

Las fuentes alimentarias de vitamina D son limitadas e incluyen fuentes naturales como pescado, yema de huevo y alimentos fortificados como la leche y los cereales (tabla 2) ${ }^{(2)}$. Sin embargo, sus concentraciones son tan bajas que difícilmente se llegaría a la ingesta diaria recomendada.

Tabla 2. Fuentes de vitamina D

\begin{tabular}{l|l}
\hline Fuente & Contenido de vitamina \\
\hline Salmón fresco (3,5 oz) & $600-1000$ UI vitamina D3 \\
\hline Sardinas enlatadas $(3,5 \mathrm{oz})$ & 300 UI vitamina D3 \\
\hline Atún enlatado $(3,5 \mathrm{oz})$ & 250 UI vitamina D3 \\
\hline Yema de huevo & 20 UI vitamina D 2 \\
\hline Leche fortificada & 100 UI/8 oz vitamina D3 \\
\hline Margarina fortificada & 430 UI/3.5 oz vitamina D3 \\
\hline Multivitaminas & Promedio 200-400 UI vitamina D3 \\
\hline
\end{tabular}

Adaptado de N Eng J Med 2007; 357:266-281.

La Endocrine Society en el año 2011 publicó las recomendaciones de ingesta diaria de vitamina D 3, que para el caso de las mujeres se muestran en la tabla $3^{(41)}$.

Una situación diferente es la dosis en caso de deficiencia $(<20 \mathrm{ng} / \mathrm{mL}$ ), la cual sería de 6000 UI diarias durante ocho
Tabla 3. Dosis diaria recomendada para mujeres Endocrine Society

\begin{tabular}{c|c|c}
\hline Grupo de edad & $\begin{array}{c}\text { Requerimiento } \\
\text { diario }\end{array}$ & UL \\
\hline $9-18$ años & $600-1.000 \mathrm{UI}$ & $4.000 \mathrm{UI}$ \\
\hline $19->70$ años & $1.500-2.000 \mathrm{UI}$ & $10.000 \mathrm{UI}$ \\
\hline
\end{tabular}

UL, Límite máximo tolerable de ingesta. Fuente: J Clin Endocrinol Metab. 2011, 96(7): 1911-1930

semanas, para luego continuar con 1.500 a 2.000 UI/día de sostenimiento. Tratando de mantener los niveles de 25- $(\mathrm{OH})$ D mayor de $30 \mathrm{ng} / \mathrm{mL}^{(41)}$.

\section{Intoxicación por vitamina $D$}

La intoxicación por vitamina D es extremadamente rara, ya que la retroalimentación por 1,25(OH) 2 D3 limita los niveles circulantes, minimizando la intoxicación potencial por vitamina D (3); sin embargo, esta puede ocurrir por ingesta inadvertida de dosis excesivamente altas.

Dosis de más de 50.000UI/día de vitamina D 3, elevan los niveles de 25-(OH) D por encima de $150 \mathrm{ng} / \mathrm{ml}$ y se asocian con hipercalcemia e hiperfosfatemia ${ }^{(42)}$.

\section{Conclusiones}

El déficit de vitamina $\mathrm{D}$ se constituye en una epidemia de alcance mundial. Anteriormente se consideraba que afectaba países con estaciones y baja exposición a los rayos solares; sin embargo, la evidencia actual muestra que también afecta a personas que habitan en los trópicos con adecuada exposición solar.

La vitamina D es fundamental para la adecuada salud ósea y recientes publicaciones han demostrado su efecto, no sólo sobre marcadores subrogados de salud esquelética sino también sobre el riesgo de fractura.

Actualmente se cuenta con buenas pruebas comerciales para la medición de 25-(OH) D, la vigilancia del estatus sérico de vitamina D debe formar parte integral del estudio de la osteoporosis en mujeres posmenopáusicas y debemos familiarizarnos con esta prueba.

La evidencia sugiere que la dosis diaria recomendada actualmente de vitamina D 3 debe ser de 800 UI por día, dado el bajo aporte suministrado por la dieta y el riesgo de quemaduras y cáncer de piel asociado a la exposición solar, sería prudente recomendar a las mujeres posmenopáusicas mejorar el aporte de vitamina D a través de suplementos orales. 


\section{Referencias}

1. Holick MF. Vitamin D: Photobiology, metabolism, mechanism of action, and clinical applications. Flavus M. editor. Primer on the Metabolic Bone Diseases and Disorders of Mineral Metabolism. American society for Bone and Mineral Research. Washington D.C. 2003; 129-137.

2. Holick MF. Vitamin D Deficiency. N Engl J Med 2007; 357:266-281.

3. Dusso A, Brown J, Slatopolsky E. Vitamin D. Am J Renal Physiol; 2005; 289: F8-F28.

4. Bouillon R. Vitamin D From photosyntesis, metabolism and action to clinical applications. De Groot LJ, Jamerson LJ. editors. Endocrinology. Philadelphia; WB Saunders; 2001; 1009-1035.

5. Kurnik B, Hruska K. Mechanism of stimulation of renal phosphate transport by 1,25-dihydroxycholecaiciferol. Biochim Biophys Acta; 1985; 817: 42-50.

6. Lips P. Vitamin D Deficiency and Secondary Hyperparathyroidism in the Elderly: Consequences for Bone Loss and Fractures and Therapeutic Implications. Endocrine Reviews; 2001; 22(4): 477-501.

7. Stejskal D, Bartek J, Pastrokova R, et al. Osteoprotegerin, RANK, RANKL. Biomed Papers; 2001; 145:61-64.

8. Lips P. The effect of vitamin D supplementation on vitamin D status and Parathyroid Function in elderly subjects. J Clin Endocrinol Metab; 1988; 67: 644-650.

9. Bruce D' St John A' Nicklason F' et al. Secondary Hyperparathyroidism in Patients from Western Australia with Hip Fracture: Relationship to Type of Hip Fracture, Renal Function, and Vitamin D Deficiency. J American Geriatrics Society; 1999; 47 (3): 354-359.

10. Chapuy MC, Preziosi P, Maamer M, et al. Prevalence of Vitamin D Insufficiency in an Adult Normal Population. Osteoporos Int; 1997; 7:439-443.

11. Okumus M, Koybası M, Tuncay F, et al Fibromyalgia syndrome: is it related to vitamin D deficiency in premenopausal female patients? Pain Manag Nurs. 2013 Dec;14(4):e156-161.

12. Jesus CA, Feder D, Peres MF. The role of vitamin D in pathophysiology and treatment of fibromyalgia. Curr Pain Headache Rep. 2013; 17(8):355-362.

13. Guilsun Kim, Ki Won Oh, Eun-Hee Jang et al. Relationship between Vitamin D, Parathyroid Hormone, and Bone Mineral Density in Elderly Koreans. J Korean Med Sci 2012; 27: 636-643.

14. Le Boff MS, Kohlmeier L, Hurwitz S. Occult vitamin D deficiency in postmenopausal US woman with acute hip fracture. JAMA; 1999; 281:1505-1511.

15. Kristin Holvik, Luai A. Ahmed, Siri Forsmo. Low Serum Levels of 25-Hydroxyvitamin D Predict Hip Fracture in the Elderly: A NOREPOS Study J Clin Endocrinol Metab 2013 98:8, 3341-3350.

16. Alfonso J. Cruz-Jentoft1, Jean Pierre Baeyens, Jürgen M. Bauer et al Sarcopenia: European consensus on definition and diagnosisReport of the European Working Group on Sarcopenia in Older People. Age Ageing 2010: 39 (4): 412-423.

17. Holick MF. High prevalence of vitamin D inadequacy and implications for Health. Mayo Clin Proc; 2006; 81: 353-373.

18. Christian M. Girgis, Roderick J. Clifton-Bligh et al. The Roles of Vitamin D in Skeletal Muscle: Form, Function, and Metabolism. Endocr Rev. 2013 Feb;34(1):33-83.

19. Dawson H, Heaney RP, Holick MF. Estimation of optimal vitamin D status. Osteoporos Int; 2005; 16: 713-716.

20. Priemel M. Von Domarus C, Klatte TO. Bone mineralization defects and vitamin D deficiency: Histomorphometric analysis of iliac crest bone biopsies and circulating 25-hydroxyvitamin D in 675 patients. J Bon Miner Res 2010; $25,2,: 305-312$.

21. Holick MF, Siris E, Binkley N. Prevalence of Vitamin D Inadequacy among Postmenopausal North American Women Receiving Osteoporosis Therapy. J Clin Endocrinol Metab; 2005; 90: 3215-3224.

22. Nesby-O'Dell S, Scanlon K, Cogswell M. et al. Hypovitaminosis D prevalence and determinants among African American and white women of reproductive age. Am J Clin Nutr; 2002; 76: 187-192.

23. Rodríguez J, Valdivia G, Trincado P. Fracturas vertebrales osteoporosis y vi- tamina D en la postmenopausia. Estudio en 555 mujeres en Chile. Rev Med Chile; 2007; 135: 31-36.

24. Binkley N, Novotny R, Krueger D. Low Vitamin D Status despite Abundant Sun Exposure. J Clin Endocrinol Metab; 2007; 92: 2130-2135.

25. Deyanira, González Devia; Claudia, Zúñiga Libreros; William, Kattah Calderón. Insuficiencia de vitamina $D$ en pacientes adultos con baja masa ósea y osteoporosis en la Fundación Santa Fe de Bogotá 2008-2009 / Vitamin D insufficiency in adults patients with low bone mass and osteoporosis in the Fundación Santa Fe de Bogotá 2008-2009. Rev.Colomb.Reumatol.; 17(4); 212-218; 2010-09

26. Molina JF, Molina J, Jorge, Escobar JA. et al Niveles de 25 hidroxivitamina D y su correlación clínica con diferentes variables metabólicas y cardiovasculares en una población de mujeres posmenopáusicas. Acta Med Colomb 2011; 36: 18-23.

27. María Patricia, Hormaza; Diana, Cuesta; Lina María, Martínez; Mónica María, Massaro; María Nazareth, Campo; María del Pilar, Vélez; Gabriel Jaime, Rendón; Camilo Andrés, Agudelo. Niveles séricos de 25 hidroxivitamina d en mujeres no menopáusicas, menopáusicas y posmenopáusicas. Rev Colomb Obstet Ginecol; 62(3); 231-236; 2011-09.

28. Chel VGM, Ooms ME, Pooo-Snijders L, et al. Ultraviolet Irradiation Corrects Vitamin D Deficiency and Suppresses Secondary Hyperparathyroidism in the Elderly. J Bone Miner Res; 1998; 13: 1238-1242.

29. The American Academic of dermatology. Position Statement on Vitamin D.http://www.aad.org/Forms/Policies/Uploads/PS/PSVitamin\%20D\%20 Postition\%20Statement.pdf

30. Brazier M, Kamel S, Maamer M. et al. Markers of bone remodelling in the elderly subject: effects of vitamin d insufficiency and its correction. J Bone Miner Res; 1995; 10: 1753-1761.

31. Prestwood KM, Pannullo AM, Kenny AM, et al. The effect of a short course of calcium and vitamin D on bone turnover in older women. Osteoporos Int; 1996; 6:314-319.

32. Sakalli H, Arslan D, Yucel AE. The efect of oral and parenteral vitamin D supplementation in the elderly: a prospective, double-blinded, randomized, placebo-controlled study.; Rheumatol Int; 2012: 32:2279-2283.

33. Grimnes G, Joakimsen R, Figenschau Y, Torjesen PA, Almås B, Jorde R. The effect of high-dose vitamin $\mathrm{D}$ on bone mineral density and bone turnover markers in postmenopausal women with low bone mass--a randomized controlled 1-year trial. Osteoporos Int. 2012: 23(1):201-11.

34. Ooms M, Roos J, Bezemer P, et al. Prevention of bone loss by Vitamin D supplementation in elderly women: a randomized double-blind trial. J Clin Endocrinol Metab; 1995; 80:1052-1058.

35. Reid IR, Bolland MJ, Grey A. Effects of vitamin D supplements on bone mineral density: a systematic review and meta-analysis. Lancet. 2014 11;383 (9912):146-55.

36. Boonen S, Bischoff F, Cooper C, et al. Addressing the musculoskeletal components of fracture risk with calcium and vitamin D: a review of the evidence. Calcif Tissue Int; 2006; 78: 257-270.

37. Murad MH, Elamin KB, Abu Elnour NO, Elamin MB, Alkatib AA, Fatourechi MM et al. Clinical review: The effect of vitamin D on falls: a systematic review and meta-analysis. J Clin Endocrinol Metab. 2011; 96(10):2997-3006

38. Kattah W. Epidemiología de osteoporosis algunos datos de la población mundial. Revista Colombiana de Menopausia; 1995; 1 (2) 33-35.

39. Bischoff $\mathrm{H}$, Willett $\mathrm{W}$, Wong J, et al. Fracture prevention With vitamin $\mathrm{D}$ supplementation. JAMA; 2005; 293; 2257-2264.

40. Heike A. Bischoff F, Walter C. Willett, E,Lips P, Meunier PJ, Ronan A. et al. A Pooled Analysis of Vitamin D Dose Requirements for Fracture Prevention. N Engl J Med 2012; 367:40-49.

41. Holick MF, Binkley N, Bischoff-Ferrari, Gordon CM, Hanley DA, Heaney RP, et al. Evaluation, Treatment, and Prevention of Vitamin D Deficiency: An Endocrine Society Clinical Practice Guideline. J Clin Endocrinol Metab. 2011, 96(7):1911-1930.

42. Fahad Alshahrani, Naji Aljohani. Vitamin D: Deficiency, Sufficiency and Toxicity. Nutrients. 2013; 5 (9):3605-3616. 